\title{
Abnormal function of monoamine oxidase-A in comorbid major depressive disorder and cardiovascular disease: Pathophysiological and therapeutic implications (Review)
}

\author{
RODRIGO MACHADO-VIEIRA ${ }^{1}$ and ALAN G. MALLINGER ${ }^{2,3}$ \\ ${ }^{1}$ Laboratory of Neuroscience, LIM27, Institute and Department of Psychiatry, University of Sao Paulo, \\ SP, Brazil; ${ }^{2}$ National Institute of Mental Health, National Institutes of Health, Bethesda, MD 20892; \\ ${ }^{3}$ Office of Inspector General, Department of Veterans Affairs, Washington, DC, USA
}

Received May 21, 2012; Accepted August 16, 2012

DOI: $10.3892 / \mathrm{mmr} .2012 .1062$

\begin{abstract}
The association between major depressive disorder (MDD) and cardiovascular disease (CVD) is among the best described medical comorbidities. The presence of MDD increases the risk of cardiac admissions and mortality and increases healthcare costs in patients with CVD, and similarly, CVD affects the course and outcome of MDD. The potential shared biological mechanisms involved in these comorbid conditions are not well known. However, the enzyme monoamine oxidase-A (MAO-A), which has a key role in the degradation of catecholamines, has been associated with the pathophysiology and therapeutics of both MDD and CVD. Increased MAO-A activity results in the dysregulation of downstream targets of this enzyme and thus affects the pathophysiology of the two diseases. These deleterious effects include altered noradrenaline turnover, with a direct elevation in oxidative stress parameters, as well as increased platelet activity and cytokine levels. These effects were shown to be reversed by MAO inhibitors. Here, a model describing a key role for the MAO-A in comorbid MDD and CVD is proposed, with focus on the shared pathophysiological mechanisms and the potential therapeutic relevance of agents targeting this enzyme.
\end{abstract}

Correspondence to: Dr Rodrigo Machado-Vieira, Laboratory of Neuroscience, LIM27, Institute and Department of Psychiatry, University of Sao Paulo, Rua Ovidio Pires de Campos 785, 01060-970 Sao Paulo, SP, Brazil

E-mail: rvieira@usp.br

Abbreviations: MDD, major depressive disorder; CVD, cardiovascular disease; MI, myocardial infarction; 5-HIAA, 5-hydroxyindoleacetic acid; 5-HT, serotonin; NE, norepinephrine; DA, dopamine; $\mathrm{NH}_{3}$, ammonia; $\mathrm{H}_{2} \mathrm{O}_{2}$, hydrogen peroxide; MAO-A, monoaminoxidase-A

Key words: depression, cardiovascular disease, MAO, noradrenaline, comorbidity, oxidative stress, inflammation

\section{Contents}

1. Introduction

2. The role of monoamine oxidase-A in the pathophysiology and therapeutics of MDD

3. The role of MAO-A in the pathophysiology and therapeutics of CVD

4. Increased MAO-A activity as a factor linking depression with comorbid cardiac disease

5. Conclusions and perspectives

\section{Introduction}

Comorbid major depressive disorder and cardiovascular disease: general aspects. Major depressive disorder (MDD) is a serious, recurrent, prevalent and disabling psychiatric illness affecting millions of individuals worldwide, which presents a negative impact on medical health and productivity (1). Similarly, an estimated 80 million Americans have at least one form of cardiovascular disease (CVD) (2).

The association between MDD and CVD is among the best described medical comorbidities (3). Early epidemiological studies showed that the age-adjusted mortality rate in depression was approximately 6 times higher than in the general population, with almost half of these cases directly related to 'diseases of the heart' (4). Recent studies showed that around $1 / 5$ of subjects recently diagnosed with CVD also have MDD (5-7). Despite being well documented, MDD remains underdiagnosed in patients with CVD and presents a direct negative impact on the course and outcome of CVD (8). For instance, the presence of depressive symptoms significantly enhances the risk of cardiac admissions and mortality, also increasing healthcare costs in patients with a cardiac disease (9).

Furthermore, according to a recent survey, $50 \%$ of cardiologists were unaware of MDD as an independent risk factor for CVD and $71 \%$ address the potential presence of MDD in less than half their patients with CVD. In total, $79 \%$ do not use any standard screening method to diagnose depression (10). Thus, the assessment of this comorbidity in clinical practice and the study of its potential biological variables may 
potentially help in the development of new, improved treatments able to treat the two conditions concomitantly.

Comorbid MDD and CVD: is there a primary and secondary condition? More than 100 studies have evaluated the potential association between MDD and CVD, showing that depression is more prevalent (20-35\%) in populations with any CVD (11). Clearly, MDD has been associated with an increased incidence of cardiac disease and worsens the prognosis in patients with known coronary heart disease (e.g., doubling the risk of cardiac events) (9). Recently, Ellis et al (12) described that $41.2 \%$ of 490 subjects with an acute coronary event present depressive symptoms, while only $10 \%$ of them were adequately treated with antidepressants and/or psychosocial support.

It has been proposed that the majority of coronary disease follows rather than precedes MDD, but it may be considered that previous environmental and/or genetic factors can lead to biological changes in the brain and periphery that may give rise to the two conditions (13). By contrast, certain authors suggest that MDD induces specific biological changes centrally that could increase the risk of CVD (13). Another possibility is that the association of MDD and CVD is indirect. For example, there is evidence that depressed patients are less likely to comply with treatments for cardiovascular conditions (14). It has also been proposed that altered circadian rhythms described in mood disorders may disrupt cardiovascular physiology, such as heart rate and blood pressure, thus increasing the risk for adverse cardiac events, such as heart attack and stroke (15).

Evidence for $M D D$ as a primary condition. Convincing evidence has suggested a key role for MDD in the onset and course of CVD. First, the risk of CVD is clearly higher in subjects with MDD (16). Diverse meta-analyses have described effect sizes for MDD in the development of coronary heart disease from $\sim 1.5$ to $2.7(3,17-19)$. For instance, subjects presenting a depressive episode at the time of an acute myocardial infarction (MI) have a significant increase in mortality rates compared to those not in a depressive episode (20). Specifically, changes in depressive symptoms (21) or a new episode of MDD (22) enhance the risk of coronary events rather than chronic depressive symptoms. Depressive symptoms such as insomnia have also been associated with increased risk of MI (23) and cardiac mortality (24). These effects appear to involve several pathophysiological effects in MDD that may be directly connected to the arousal of adverse cardiac outcomes $(25,26)$.

Evidence for CVD as a primary condition. MDD has been considered an independent risk factor for CVD (20). In patients with coronary heart disease, size effects for the prediction of MDD range between 1.6 and $2.2(18,27,28)$. Notably, it was shown that following MI, more than $20 \%$ of all patients met criteria for MDD (29). Regarding potential indirect associations, many cardiovascular drugs are capable of inducing depressive symptoms, such as $\beta$-blockers, methyldopa and reserpine (30).

Overall, the evidence supports a role for both MDD and CVD as the primary condition in this comorbidity, with more consistent data for MDD increasing risk for CVD.

\section{The role of monoamine oxidase-A in the pathophysiology and therapeutics of MDD}

Monoamine oxidases: general aspects. Monoamine oxidases (MAOs) are mitochondrial flavoenzymes that catalyze oxidative deamination of dietary amines, monoamine neurotransmitters and hormones, including indoleamines [serotonin (5-HT) and tryptamine] and catecholamines, such as norepinephrine (NE), epinephrine and dopamine (DA) (31). Thus, MAO is responsible for the metabolism of biologically active amines. The process of oxidative deamination of these amines results in removal of the amino functional group to leave an oxidized oxygen, thus generating the toxic products ammonia $\left(\mathrm{NH}_{3}\right)$ and hydrogen peroxide $\left(\mathrm{H}_{2} \mathrm{O}_{2}\right)(32) . \mathrm{H}_{2} \mathrm{O}_{2}$ is a key mediator in the production of the most potent free oxygen radicals, namely the hydroxyl radical $(\mathrm{OH} \cdot)$ (33), which induces deleterious effects in several organs, particularly the brain.

Two isoforms of MAO have been identified, designated types $\mathrm{A}$ and $\mathrm{B}$, which have distinct substrate affinity and inhibitor sensitivity (34). MAO-A is the major form of this enzyme found in the periphery. In the brain, MAO-A is mostly found in outer mitochondrial membranes in noradrenergic neurons, while MAO-B has been observed in glial cells and 5-HT/histaminergic neurons (35). The ratio of MAO-A to MAO-B in the human brain is $25 / 75 \%$, even though MAO-A inhibitors have shown superior antidepressant effects (36). In fact, inhibition of MAO-A is thought to be the action most directly linked with the antidepressant activity of the MAO inhibitors (37). MAO-A preferentially metabolizes NE and 5-HT, the monoamines most closely linked to depression, while MAO-B preferentially metabolizes trace amines, including phenethylamine (38). The degradation of biogenic amines by $\mathrm{MAO}-\mathrm{A}$ has been considered the major physiological function of this enzyme.

MAO-A in the pathophysiology and therapeutics of MDD. The primary role of MAO-A is to regulate monoaminergic turnover and levels. Elevated MAO-A levels may be expected to metabolize NE and 5-HT more extensively (34), thus resulting in relative monoamine depletion.

This effect may be critical in the pathophysiology of MDD. MAO-A regulates the levels of NE by catalyzing its oxidative deamination (31). The increased sympathoadrenal system activity observed in MDD has been associated with enhanced excretion of NE, epinephrine and dopamine (39). Similarly, patients with MDD are reported to have increased plasma NE levels, increased heart rates and reduced heart-rate variability (40). Regarding the potential role of MAO-A in the pathophysiology of MDD, it was recently described that unmedicated subjects with MDD show greater depletion of previously synthesized stores of 5-HT, along with higher levels of 5-hydroxyindoleacetic acid (5-HIAA) (41). 5-HIAA is produced by intraneuronal deamination of 5-HT (via MAO-A).

In regard to the 5-HT regulation by MAO-A, in the tryptophan depletion challenge, mood lowering effects were more prevalent in unmedicated euthymic subjects with a history of MDD as compared to healthy controls (42-45). Higher MAO-A levels may explain the increased vulnerability to tryptophan depletion in MDD patients in recovery through excessive metabolism of 5-HT by MAO-A, which would facilitate 
loss of extracellular 5-HT. By contrast, selective serotonin reuptake inhibitors (SSRIs) decrease 5-HIAA production by blocking neuronal 5-HT reuptake, which elevates 5-HT levels substantially (46). Notably, SSRIs were also shown to reverse different dysfunctions associated with the pathophysiology of MDD and CVD, such as altered urinary cortisol excretion and heart rate variability, as well as enhanced platelet activation and increased inflammatory marker levels (47-49).

Since MAO-A is involved in the removal of multiple monoamines, increased MAO-A binding in MDD may be involved in the potential mood-lowering effects following depletion of $\mathrm{NE}$ with $\alpha$-methylparatyrosine administration $(50,51)$, as well as having a potential association with increased risk of recurrence of MDD (52).

Increased MAO-A density in several brain areas has been considered an important monoamine-lowering process during depressive episodes in MDD $(52,53)$. A previous PET study using carbon 11-labeled harmine (a tracer with high affinity to MAO-A) showed an abnormal increase in MAO-A binding during depressive episodes (53). A subsequent PET study evaluated 28 healthy subjects, 16 subjects with MDD in a depressive episode and 18 subjects with MDD in recovery prior to and following 6 weeks of SSRI treatment, followed up for 6 months after MAO-A binding quantification. The authors demonstrated a significant increase in MAO-A density during a depressive episode compared with healthy controls, which remained elevated following treatment with SSRIs (52). Although brain MAO-A density was generally enhanced during recovery, patients who experienced depressive recurrence showed significantly higher MAO-A density in the prefrontal and anterior cingulate cortex as compared to those who did not (52). Based on these findings, it was proposed that higher MAO-A binding (and density) may be considered a trait marker in MDD. It was also found that the regional density of MAO-A transporters has a selective influence on particular monoamines, with a direct association with specific clinical presentation (53). A recent study showed that the MAO-A total distribution volume was significantly elevated (by a mean of $43 \%$ ) in different brain regions during the early postpartum period, indicating that this monoamine-lowering process contributes to the mood change in postpartum blues (54).

Given that an abnormal increase in MAO-B density is less expected to occur in MDD, the present results appear to be specific to MAO-A. For instance, a postmortem study described no significant difference of MAO-B density in the amygdala of subjects with MDD (55).

Regarding genetic studies, MAO-A (located at Xp11.3) has been considered an important candidate gene in mood disorders. Besides being considered as a potential risk gene in depressed suicidal patients (56), it has been associated with specific psychological traits (57). Studies also suggest that MAO-A gene alleles associated with higher transcriptional efficiency predispose to dysfunctional behavior such as trait aggressiveness and impulsivity $(58,59)$. Three common polymorphisms that critically affect transcriptional efficiency have been evaluated in association studies on the MAO-A gene in mood disorders: i) a promoter variable number tandem repeat polymorphism (uVNTR) $(60,61)$; ii) a G/T polymorphism at position 941 of the cDNA sequence, which is a silent mutation in exon 8 (62); and iii) a dinucleotide repeat in intron 2
(MAOA-CA) (63). Significant associations between these three common polymorphisms have been demonstrated in meta-analyses evaluating case-control association studies in MDD $(64,65)$. MAO-A knockout mice have increased brain $\mathrm{NE}$ and 5-HT levels (66). The consequent decrease in MAO-A levels improved resilience and adaptation to the effects of environmental stressors, which is also associated with antidepressant-like effects (66).

It is important to note that the MAO-A metabolism increases oxidative stress levels (34), which have been directly involved in the pathophysiology of MDD (67). The dysregulation of redox balances and mitochondrial damage induced by MAO activation may result in neuronal apoptosis and brain damage. For instance, serum-starvation-induced apoptosis increases MAO-A levels, which was prevented by using an MAO-A inhibitor in cortical neurons (68). Neurodegenerative toxicity and striatal lesions induced by malonate were significantly and selectively attenuated by MAO-A inhibitors and in MAO-A KO, without a positive response to a MAO-B inhibitor (69).

Regarding the therapeutic potential of specific MAO-A inhibitors for MDD, the clinical efficacy of diverse reversible inhibitors of MAO (including specific MAO-A inhibitors or RIMAs) has been observed in treatment-resistant depression (70), mostly related to inhibition of MAO-A (38). However, it is important to emphasize that the majority of the currently available MAO inhibitors are non-selective, inhibiting MAO-A and MAO-B (38).

\section{The role of MAO-A in the pathophysiology and thera- peutics of CVD}

Monoaminergic neurotransmitters have a critical functional role in the heart, including the regulation of cardiac inotropy (13). Increased catecholamine metabolism and altered tissue distribution regulated by MAO have been directly associated with the aging process $(71,72)$. MAO-A is present in the myocardium of diverse species from rodents to humans $(73,74)$. The heart contains a large amount of MAO-A $(75,76)$ and its role in the regulation of cardiac function critically involves NE concentrations $(77,78)$.

It was recently shown in preclinical models that $\mathrm{NE}$ is capable of triggering CVD in a MAO-A-dependent manner (79). NE catabolism and ROS production are markedly upregulated in pressure-overloaded hearts and both effects are ameliorated by limiting MAO-A activity to suppress cardiac decompensation with pressure overload (79). Similarly, increased sympathetic activity in the central nervous system (CNS) with concomitant elevated levels of catecholamines has also been proposed as one potential mechanism by which depressive symptoms may increase CVD morbidity and mortality (80). MAO-A is also an important source of hydrogen peroxide $\left(\mathrm{H}_{2} \mathrm{O}_{2}\right)$ in the heart (81). MAO-A plays a key role in reactive oxygen species-dependent cardiomyocyte apoptosis and postischemic cardiac damage (82). Elevated mitochondrial oxidative stress levels and/or decreased mitochondrial antioxidant defenses have been shown to aggravate atherosclerosis (83). MAO-A mediates reactive oxygen species (ROS)-induced activation of mitogenic signaling in endothelial cells related to vascular wall remodeling (associated with 
atherosclerosis), which were inhibited by the MAO-A inhibitors pargyline and Ro41-1049 (84).

The involvement of MAO-A and its impact on neurotransmitter availability in congestive heart failure (CHF) has also been shown. In preclinical models, left ventricular dilation and pump failure attributable to pressure overload have been associated with increased NE catabolism by MAO-A, with enhanced production of free radicals and myocardial apoptosis. MAO-A activity worsens the disease progression (13). CHF is directly associated with an increased sympathetic tone and altered oxidative stress parameters (85). In such situations, MAO-A may be upregulated, generating greater amounts of $\mathrm{H}_{2} \mathrm{O}_{2}$, and thus exacerbating disease progression. The increased oxidative stress induced by MAO-A has also been directly associated with postischemia-reperfusion apoptosis (76). Notably, inhibition of MAO-A by clorgyline ameliorated the majority of these changes (79).

The cardioprotective effects of MAO inhibitors are associated with the prevention of postischemic oxidative stress, neutrophil accumulation and mitochondrial-dependent cell death (76), thus inducing positive effects in myocardium reperfusion. The inhibition of MAO-A in vivo largely reduced myocardial ultrastructural damage following ischemia. Diverse MAO inhibitors (JB-516, JB-835, RO-50700, harmine, harmaline and iproniazid) have been shown to increase heart contractile force through effects induced by norepinephrine, dopamine, tryptamine, tyramine and serotonin in animals (86).

\section{Increased MAO-A activity as a factor linking depression with comorbid cardiac disease}

Here we describe a model integrating various pathophysiological findings and targets directly associated with the effects of MAO-A with a potential key relevance to the shared pathophysiology of comorbid MDD and CVD. Enhanced sympathetic CNS activity mediated through MAO-A is here proposed as a potential common mechanism for the development of MDD and cardiac morbidity, potentially by concomitantly increasing oxidative stress levels, activating immuno-inflammatory responses and enhancing glucocorticoid metabolism, as described below (Fig. 1).

NE activity. As mentioned previously, MAO-A critically controls brain and peripheral $\mathrm{NE}$ levels by regulating the oxidative deamination of NE (38). In preclinical models, the induction of a MAO-A-dependent NE overflow and elevated breakdown has been shown to be a key factor in the etiology of CVD; these dangerous downstream effects were stalled by using the selective MAO-A inhibitor clorgyline (79). Similarly, CVD has also been associated with alterations in autonomic balance. Elevated sympathetic tone, as measured by plasma $\mathrm{NE}$, predicts mortality in CVDs, such as left ventricular dysfunction and chronic CHF (87). Specifically, elevated NE levels in patients with $\mathrm{CHF}$ have been positively correlated with severity of symptoms and mortality. Similarly, higher NE excretion was associated with lower left ventricular ejection fraction (88). NE levels also predict mortality after MI (89). Notably, depressive symptoms in patients with CVD were associated with elevated levels of NE excretion but not DA (90). Importantly, the oxidative deamination of NE by MAO-A

\section{Comorbid Major Depressive Disorder and Cardiovascular Disease}
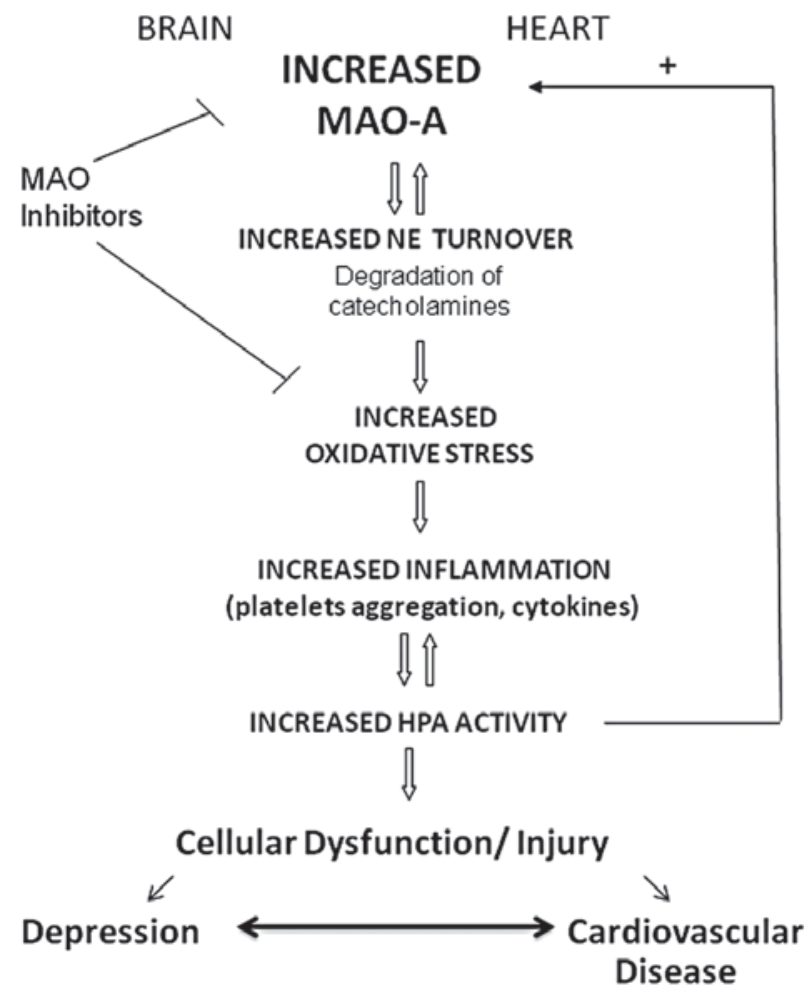

Figure 1. Integrative biological model explaining the key role for MAO-A in comorbid depression and cardiovascular disease: involvement of oxidative stress, inflammation and glucocorticoid activity. MAO-A, monoamine oxidase-A; NE, norepinephrine.

increases ROS and oxidative stress levels by releasing reactive aldehydes and $\mathrm{H}_{2} \mathrm{O}_{2}$ (as described below).

At the same time, increased sympathetic activity in the CNS with concomitant elevated levels of catecholamines turnover has also been proposed as one potential mechanism by which depressive symptoms may increase morbidity and mortality (80). The increased sympathoadrenal system activity observed in MDD has been associated with enhanced excretion of NE, epinephrine and DA (39). Patients with MDD presented with increased heart rates and reduced heart-rate variability, reflecting altered cardiac autonomic tone (40).

Oxidative stress parameters. Increased oxidative stress parameters have been described in the pathophysiology of MDD in several studies (reviewed in ref. 67). For instance, individuals in a depressive episode have significantly lower total antioxidant potential and higher oxidative stress levels compared to healthy controls (91). Diverse studies have also shown an inverse association between severity of depressive symptoms and oxidative stress levels (92-94). Increased oxidative damage and apoptosis in cortical neurons are associated with elevated MAO-A, and may be potentially prevented by using MAO-A inhibitors (68).

Similarly, CVD is directly related to increased oxidative stress levels (95). Consistent evidence from basic research 
studies suggests that reactive oxygen species contribute to atherosclerosis and CVD (96). For instance, reactive oxygen species are also capable of stimulating matrix metalloproteinases, which contribute to atherosclerotic plaque instability and rupture, thus inducing acute coronary syndromes (97). MAO-A is a recognized source of ROS $(76,79)$. These effects may involve MAO-A, since this enzyme is an important source of mitochondrial $\mathrm{H}_{2} \mathrm{O}_{2}$ in the heart, thus contributing to oxidative stress-induced cardiomyocyte apoptosis (76).

Immune-inflammatory activation. Growing evidence has shown a significant increase in immune-inflammatory markers in MDD (98), which have been directly involved in the increased production of ROS and oxidative stress levels $(99,100)$. At the same time, ROS may also induce LDL oxidation and activation of vascular smooth muscle cell proliferation and migration, as well as increase the production of proinflammatory cytokines (96). Pasic et al (101) described several findings on immune-inflammatory changes in MDD and CVD, indicating that cytokines may provide a new avenue in understanding brain-body interactions in MDD and CVD.

MAO-A activates inflammatory cascades and platelet activity, with consequent endothelial dysfunction. Platelets adhere to intact endothelial cells and promote local vascular inflammation by recruiting leukocytes via direct interaction or by activating inflammatory mediators (102). Platelets share similar biochemical processes with neurons; platelet MAO activity was found to be related to central monoamine turnover, although platelets express mainly MAO-B. Platelets are also thought to play a predominant role in the initiation and progression of atherogenesis (103). Notably, MAO-A expression was shown to be significantly increased by pro-inflammatory cytokines in human monocytes (104). MAO-A substrates, such as 5-HT and NE, may act as vasoactive mediators at inflammatory sites (104). These data support a role for MAO-A in the inflammation-inducing CVD dysfunction.

Similarly, patients with significant depressive symptomatology have altered endothelial function compared to non-depressed individuals; the use of monoaminergic antidepressants is associated with reversal of this dysfunction (105). These effects may be associated with increased platelet reactivity observed in subjects with MDD (106-108), as well as activation of inflammatory pathways in the disease (109). The association between depressive symptoms and increased platelet activity $(106,110)$ involves the metabolism of catecholamines in platelets $(106,108,111)$. Similarly, CVD has also been associated with increased platelet reactivity (112), inflammation and endothelial dysfunction (105), thus supporting a potential common etiological role for immuneinflammatory dysfunction in MDD and CVD. For instance, cytokine interleukin-10 levels predicted an adverse clinical outcome in chronic heart failure patients with depressive symptoms in a 1-year follow-up study (113). The increased activation of inflammatory cytokines and endothelial dysfunction described in the pathophysiology of MDD and CVD has been shown to involve concomitant dysfunctions in the hypothalamic-pituitary-adrenal (HPA) axis activity $(109,113)$, which has also been shown to be regulated by MAO-A (as described below).
HPA activity. Dysfunctional HPA activity, including increased glucocorticoid activity, has been described in the pathophysiology of MDD (114). It has been proposed that enhanced cortisol agonistic effects during depressive episodes may contribute to an elevation in MAO-A levels (53). Severely depressed patients had significant increases in blood pressure, CSF and plasma NE, as well as elevated plasma cortisol (115). Notably, elevated platelet MAO activity associated with increased cortisol levels has been described in depression; platelet MAO activity has also been associated with severity of depressive symptoms (116). Notably, dexamethasone administration was shown to enhance MAO-A density in the brain by 300\% (117), which supports a role for MAO-A in dysfunctional HPA axis activity described in MDD and CVD.

Similarly, dysregulation of the HPA axis and elevated cortisol levels may be a mediating factor between MDD and vulnerability to CVD. HPA axis dysregulation is also related to many CVD risk factors such as visceral obesity, hypercholesterolemia, hypertriglyceridemia, increased blood pressure, elevated heart rate and steroid-induced diabetes (118). In patients with CVD, prediction of cardiac events based on cortisol levels was directly influenced by oxidative stress parameters. Related to MAO-A activity, it was shown that NE stimulates the HPA via $\alpha$ - and $\beta$-adrenergic receptors (119). Urinary cortisol concentrations showed a positive correlation with urinary MAO-A activity (120).

\section{Conclusions and perspectives}

MDD increases the risk of cardiac mortality and morbidity in patients with CVD, and similarly CVD worsens the course and outcome of MDD, but little is known about the potential mechanisms involved in these effects. The pathophysiological findings of the association between depression and cardiac events are not consistent enough to be considered mediators, but clearly together modulate several aspects of these comorbid conditions. Reduced levels of MAO-A in the brain have been shown to induce a general increase in the resistance to the effects of environmental stressors (31), which has been critically implicated in the two conditions. Non-compliance to treatments may also represent an additional problem in this comorbidity. However, a more specific hypothesis is desirable.

Here, we describe an integrative model focusing on the effects of MAO-A in the comorbidity between MDD and CVD, based on a common mechanism. Enhanced sympathetic CNS activity and consequent increased breakdown of NE induced by excessive MAO-A activity is here proposed as a potential mechanism by which MDD increases cardiac morbidity. This dysregulation has been shown to increase production of ROS, also activating immuno-inflammatory responses and excessive glucocorticoids metabolism, which directly underlie the pathophysiology of MDD and CVD (Fig. 1). Since increased brain MAO-A appears to represent a trait marker in MDD, it is reasonable to suggest that this persistent increase may also contribute to similar changes in the periphery associated with the pathophysiology of CVD.

Further studies on the associations among family history, MAO-A polymorphisms and specific outcomes in these comorbid conditions are important. Future randomized 
controlled trials using agents potentially able to treat MDD and CVD concomitantly and reverse common dysfunctional biological factors by inhibiting MAO-A activity (e.g., RIMAs) may be considered. It is also possible that a common genetic vulnerability associated with the MAO-A gene may be involved in comorbid MDD and CVD. In this context, MAO-A activity and the potential beneficial effects of MAO-A inhibitors related to oxidative stress and antioxidant status in the brain and heart deserve further studies.

\section{Acknowledgements}

Dr Machado-Vieira would like to thank Sao Paulo Research Foundation, Brazil (Fapesp) and ABADHS (Associação Beneficente Alzira Denise Hertzog da Silva). This study was initiated at the National Institute of Mental Health, prior to Dr Mallinger's official duties with the Office of Inspector General. The views expressed in this paper do not necessarily represent the views of the Office of Inspector General, the Department of Veterans Affairs or any other agencies of the United States Government.

\section{References}

1. Kessler RC, Akiskal HS, Ames M, et al: Prevalence and effects of mood disorders on work performance in a nationally representative sample of U.S. workers. Am J Psychiatry 163: 1561-1568, 2006.

2. Lloyd-Jones DR, Adams R, Carnethon M, et al: Heart disease and stroke statistics - 2009 update: a report from the American Heart Association Statistics Committee and Stroke Statistics Subcommittee. Circulation 119: 480-486, 2009.

3. Wulsin LR and Singal BM: Do depressive symptoms increase the risk for the onset of coronary disease? A systematic quantitative review. Psychosom Med 65: 201-210, 2003.

4. Malzberg B: Mortality among patients with involution melancholia. Am J Psychiatry 93: 1231-1238, 1937.

5. Ohira T, Iso H, Satoh S, et al: Prospective study of depressive symptoms and risk of stroke among japanese. Stroke 32: 903-908, 2001 .

6. Carney RM, Saunders RD, Freedland KE, et al: Association of depression with reduced heart rate variability in coronary artery disease. Am J Cardiol 76: 562-564, 1995.

7. Glassman AH and Shapiro PA: Depression and the course of coronary artery disease. Am J Psychiatry 155: 4-11, 1998.

8. Rumsfeld JS and Ho PM: Depression and cardiovascular disease: a call for recognition. Circulation 111: 250-253, 2005.

9. Frasure-Smith N and Lesperance F: Depression and cardiac risk: present status and future directions. Postgrad Med J 86: 193-196, 2010.

10. Feinstein RE, Blumenfield M, Orlowski B, et al: A national survey of cardiovascular physicians' beliefs and clinical care practices when diagnosing and treating depression in patients with cardiovascular disease. Cardiol Rev 14: 164-169, 2006.

11. Frasure-Smith N, Lespérance F and Talajic M: Depression following myocardial infarction. Impact on 6-month survival. JAMA 270: 1819-1825, 1993.

12. Ellis JJ, Eagle KA, Kline-Regers EM, et al: Depressive symptoms and treatment after acute coronary syndrome. Int J Cardiol 99: 443-447, 2005

13. Glassman AH: Depression and cardiovascular comorbidity. Dialogues Clin Neurosci 9: 9-17, 2007.

14. Gehi AD, Haas D, Pipkin S, et al: Depression and medication adherence in outpatients with coronary heart disease: findings from the Heart and Soul Study. Arch Intern Med 165: 2508-2513, 2005 .

15. Martino TA and Sole MJ: Molecular time: an often overlooked dimension to cardiovascular disease. Circ Res 105: 1047-1061, 2009.

16. Penninx BW, Beekman AT, Honig A, et al: Depression and cardiac mortality: results from a community-based longitudinal study. Arch Gen Psychiatry 58: 221-227, 2001.
17. Rugulies R: Depression as a predictor for coronary heart disease. a review and meta-analysis. Am J Prev Med 23: 51-61, 2002.

18. Nicholson A, Kuper $\mathrm{H}$ and Hemingway H: Depression as an aetiologic and prognostic factor in coronary heart disease: a meta-analysis of 6362 events among 146538 participants in 54 observational studies. Eur Heart J 27: 2763-2774, 2006.

19. Van der Kooy K, van Hout H, Markijk H, et al: Depression and the risk for cardiovascular diseases: systematic review and meta analysis. Int J Geriatr Psychiatry 22: 613-626, 2007.

20. Bush DE, Ziegelstein RC, Tayback M, et al: Even minimal symptoms of depression increase mortality risk after acute myocardial infarction. Am J Cardiol 88: 337-341, 2001.

21. Wassertheil-Smoller S, Applegate WB, Berge K, et al: Change in depression as a precursor of cardiovascular events. SHEP Cooperative Research Group (Systoloc Hypertension in the elderly). Arch Intern Med 156: 553-561, 1996.

22. Penninx BW, Guralnik JM, Mendes de Leon CF, et al: Cardiovascular events and mortality in newly and chronically depressed persons $>70$ years of age. Am J Cardiol 81: 988-994, 1998.

23. Schwartz SW, Cornoni-Huntley J, Cole SR, et al: Are sleep complaints an independent risk factor for myocardial infarction? Ann Epidemiol 8: 384-392, 1998.

24. Mallon L, Broman JE and Hetta J: Sleep complaints predict coronary artery disease mortality in males: a 12-year follow-up study of a middle-aged Swedish population. J Intern Med 251: 207-216, 2002.

25. Rozanski A, Blumenthal JA and Kaplan J: Impact of psychological factors on the pathogenesis of cardiovascular disease and implications for therapy. Circulation 99: 2192-2217, 1999.

26. Lett HS, Blumenthal JA, Babyak MA, et al: Depression as a risk factor for coronary artery disease: evidence, mechanisms, and treatment. Psychosom Med 66: 305-315, 2004.

27. Barth J, Schumacher M and Herrmann-Lingen C: Depression as a risk factor for mortality in patients with coronary heart disease: a meta-analysis. Psychosom Med 66: 802-813, 2004.

28. Van Melle JP, De Jonge P, Spijkerman TA, et al: Prognostic association of depression following myocardial infarction with mortality and cardiovascular events: a meta-analysis. Psychosom Med 66: 814-822, 2004.

29. Thombs BD, Bass EB, Ford DE, et al: Prevalence of depression in survivors of acute myocardial infarction. J Gen Intern Med 1: 30-38, 2006.

30. Huffman JC and Stern TA: Neuropsychiatric consequences of cardiovascular medications. Dialogues Clin Neurosci 9: 29-45, 2007.

31. Bortolato M, Chen K and Shih JC: Monoamine oxidase inactivation: from pathophysiology to therapeutics. Adv Drug Deliv Rev 60: 1527-1533, 2008.

32. Alper G, Girgin FK and Ozgonul M: MAO inhibitors and oxidant stress in aging brain tissue. Eur Neuropsychopharmacol 9: 247-252, 1999.

33. Cheeseman KH and Slater TF: An introduction to free radical biochemistry. Br Med Bull 49: 481-493, 1993.

34. Youdim MB and Bakhle YS: Monoamine oxidase: isoforms and inhibitors in Parkinson's disease and depressive illness. Br J Pharmacol 147: S287-S296, 2006.

35. Levitt P, Pintar JE and Breakfield XO: Immunocytochemical demonstration of monoamine oxidase $\mathrm{B}$ in brain astrocytes and serotonergic neurons. Proc Natl Acad Sci USA 79: 6385-6389, 1982.

36. Patkar AA, Pae CU and Masand PD: Transdermal selegiline: the new generation of monoamine oxidase inhibitors. CNS Spectr 11: 363-375, 2006.

37. Krishnan KR: Revisiting monoamine oxidase inhibitors. J Clin Psychiatry 68: 35-41, 2007.

38. Stahl SM and Felker A: Monoamine oxidase inhibitors: a modern guide to an unrequited class of antidepressants. CNS Spectr 13: 855-870, 2008.

39. Maes M, Meltzer HY, Suy E, et al: Sleep disorders and anxiety as symptom profiles of sympathoadrenal system hyperactivity in major depression. J Affect Disord 27: 197-207, 1993.

40. Jiang W, Krishnan RR and O'Connor CM: Depression and heart disease: evidence of a link, and its therapeutic implications. CNS Drugs 16: 111-127, 2002.

41. Barton DA, Esler MD, Dawood T, et al: Elevated brain serotonin turnover in patients with depression: effect of genotype and therapy. Arch Gen Psychiatry 65: 38-46, 2008.

42. Leyton M, Young SN, Blier P, et al: The effect of tryptophan depletion on mood in medication-free, former patients with major affective disorder. Neuropsychopharmacology 16: 294-297, 1997. 
43. Moreno FA, Gelenberg AJ, Heninger GR, et al: Tryptophan depletion and depressive vulnerability. Biol Psychiatry 46: 498-505, 1999.

44. Neumeister A, Nugent AC, Waldeck T, et al: Neural and behavioral responses to tryptophan depletion in unmedicated patients with remitted major depressive disorder and controls. Arch Gen Psychiatry 61: 765-773, 2004.

45. Ruhe HG, Mason NS and Schene AH: Mood is indirectly related to serotonin, norepinephrine and dopamine levels in humans: a meta-analysis of monoamine depletion studies. Mol Psychiatry 12: 331-359, 2007

46. Bel N and Artigas F: Chronic treatment with fluvoxamine increases extracellular serotonin in frontal cortex but not in raphe nuclei. Synapse 15: 243-245, 1993.

47. Glassman AH, O'Connor CM, Califf RM, et al: Sertraline treatment of major depression in patients with acute MI or unstable angina. JAMA 288: 701-709, 2002.

48. Tuglu C, Kara SH, Caliyurt O, et al: Increased serum tumor necrosis factor-alpha levels and treatment response in major depressive disorder. Psychopharmacology (Berl) 170: 429-433, 2003.

49. Serebruany VL, Glassman AH, Malinin AI, et al: Enhanced platelet/endothelial activation in depressed patients with acute coronary syndromes: evidence from recent clinical trials. Blood Coagul Fibrinolysis 14: 563-567, 2003.

50. Berman RM, Sanacora G, Anand A, et al: Monoamine depletion in unmedicated depressed subjects. Biol Psychiatry 51: 469-473, 2002.

51. Hasler G, Fromm S, Carlson PJ, et al: Neural response to catecholamine depletion in unmedicated subjects with major depressive disorder in remission and healthy subjects. Arch Gen Psychiatry 65: 521-531, 2008.

52. Meyer JH, Wilson AA, Sagrati S, et al: Brain monoamine oxidase A binding in major depressive disorder: relationship to selective serotonin reuptake inhibitor treatment, recovery, and recurrence. Arch Gen Psychiatry 66: 1304-1312, 2009.

53. Meyer JH, Ginovart N, Boovariwala A, et al: Elevated monoamine oxidase a levels in the brain: an explanation for the monoamine imbalance of major depression. Arch Gen Psychiatry 63: 1209-1216, 2006.

54. Sacher J, Wilson AA, Houle S, et al: Elevated brain monoamine oxidase A binding in the early postpartum period. Arch Gen Psychiatry 67: 468-474, 2010.

55. Karolewicz B, Klimek V, Zhu H, et al: Effects of depression, cigarette smoking, and age on monoamine oxidase B in amygdaloid nuclei. Brain Res 1043: 57-64, 2005.

56. Du L, Faludi G, Palkovits M, et al: High activity-related allele of MAO-A gene associated with depressed suicide in males. Neuroreport 13: 1195-1198, 2002.

57. Shih JC, Chen K and Ridd MJ: Monoamine oxidase: from genes to behavior. Annu Rev Neurosci 22: 197-217, 1999.

58. Manuck SB, Flory JD, Ferrell RE, et al: A regulatory polymorphism of the monoamine oxidase-A gene may be associated with variability in aggression, impulsivity, and central nervous system serotonergic responsivity. Psychiatry Res 95: 9-23, 2000.

59. Manor I, Tyano S, Mel E, et al: Family-based and association studies of monoamine oxidase A and attention deficit hyperactivity disorder (ADHD): preferential transmission of the long promoter-region repeat and its association with impaired performance on a continuous performance test (TOVA). Mo Psychiatry 7: 626-632, 2002.

60. Sabol SZ, Hu S and Hamer D: A functional polymorphism in the monoamine oxidase A gene promoter. Hum Genet 103: 273-279, 1998.

61. Deckert J, Catalano M, Syagailo YV, et al: Excess of high activity monoamine oxidase A gene promoter alleles in female patients with panic disorder. Hum Mol Genet 8: 621-624, 1999.

62. Lim LC, Powell J, Sham P, et al: Evidence for a genetic association between alleles of monoamine oxidase A gene and bipolar affective disorder. Am J Med Genet 60: 325-331, 1995.

63. Black GC, Chen ZY, Craig IW, et al: Dinucleotide repeat polymorphism at the MAOA locus. Nucleic Acids Res 19: 689, 1991.

64. Fan M, Liu B, Jiang T, et al: Meta-analysis of the association between the monoamine oxidase-A gene and mood disorders. Psychiatr Genet 20: 1-7, 2010.

65. Brummett BH, Krystal AD, Siegler IC, et al: Associations of a regulatory polymorphism of monoamine oxidase-A gene promoter (MAOA-uVNTR) with symptoms of depression and sleep quality. Psychosom Med 69: 396-401, 2007.
66. Cases O, Seif I, Grimsby J, et al: Aggressive behavior and altered amounts of brain serotonin and norepinephrine in mice lacking MAOA. Science 268: 1763-1766, 1995.

67. Ng F, Berk M, Dean O, et al: Oxidative stress in psychiatric disorders: evidence base and therapeutic implications. Int J Neuropsychopharmacol 11: 851-876, 2008.

68. Ou X, Chen MK and Shih JC: Monoamine oxidase A and repressor R1 are involved in apoptotic signaling pathway. Proc Natl Acad Sci USA 103: 10923-10928, 2006.

69. Maragos WF, Young KL, Altman CS, et al: Striatal damage and oxidative stress induced by the mitochondrial toxin malonate are reduced in clorgyline-treated rats and MAO-A deficient mice. Neurochem Res 29: 741-746, 2004.

70. Papakostas GI and Fava M: A metaanalysis of clinical trials comparing moclobemide with selective serotonin reuptake inhibitors for the treatment of major depressive disorder. Can J Psychiatry 51: 783-790, 2006

71. Oreland L and Gottfries CG: Brain and brain monoamine oxidase in aging and in dementia of Alzheimer's type. Prog Neuropsychopharmacol Biol Psychiatry 10: 533-540, 1986.

72. Kalaria RN, Mitchell MJ and Harik SI: Monoamine oxidases of the human brain and liver. Brain 111: 1441-1451, 1988 .

73. Rodriguez MJ and Saura J: Cellular localization of monoamine oxidase A and B in human tissues outside of the central nervous system. Cell Tissue Res 304: 215-220, 2001

74. Saura J, Kettler R, De Prada M, et al: Quantitative enzyme radioautography with $3 \mathrm{H}-\mathrm{Ro} 41-1049$ and $3 \mathrm{H}-\mathrm{Ro}$ 19-6327 in vitro: localization and abundance of MAO-A and MAO-B in rat CNS peripheral organs, and human brain. J Neurosci 12: 1977-1999, 1992.

75. Sivasubramaniam SD, Finch CC, Rodrgiguez MJ, et al: A comparative study of the expression of monoamine oxidase-A and -B mRNA and protein in non-CNS human tissues. Cell Tissue Res 313: 291-300, 2003

76. Bianchi P, Kunduzova O, Masini E, et al: Oxidative stress by monoamine oxidase mediates receptor-independent cardiomyocyte apoptosis by serotonin and postischemic myocardial injury. Circulation 112: 3297-3305, 2005.

77. Raasch W, Bartels T, Gieselberg A, et al: Angiotensin I-converting enzyme inhibition increases cardiac catecholamine content and reduces monoamine oxidase activity via an angiotensin type 1 receptor-mediated mechanism. J Pharmacol Exp Ther 300: 428-434, 2002

78. Lamontagne D, Yamaguchi N, Ribout C, et al: Reduction of tissue noradrenaline content in the isolated perfused rat heart during ischemia: importance of monoamine oxidation. Can J Physiol Pharmacol 69: 1190-1195, 1991.

79. Kaludercic N, Takimoto E, Nagayama T, et al: Monoamine oxidase A-mediated enhanced catabolism of norepinephrine contributes to adverse remodeling and pump failure in hearts with pressure overload. Circ Res 106: 193-202, 2010.

80. Carney RM and Freedland KE: Depression, mortality, and medical morbidity in patients with coronary heart disease. Biol Psychiatry 54: 241-247, 2003.

81. Maurel A, Hernandez C, Kunduzova O, et al: Age-dependent increase in hydrogen peroxide production by cardiac monoamine oxidase A in rats. Am J Physiol Heart Circ Physiol 284: 1460-1467, 2003.

82. Pchejetski D, Kunduzova O, Dayon A, et al: Oxidative stressdependent sphingosine kinase-1 inhibition mediates monoamine oxidase A-associated cardiac cell apoptosis. Circ Res 100: 41-49, 2007.

83. Halaris A: Comorbidity between depression and cardiovascular disease. Int Angiol 28: 92-99, 2009.

84. Coatrieux C, Sanson M, Negre-Salvayre A, et al: MAO-Ainduced mitogenic signaling is mediated by reactive oxygen species, MMP-2, and the sphingolipid pathway. Free Radic Biol Med 43: 80-89, 2007.

85. Takimoto E, Champion HC, Li M, et al: Oxidant stress from nitric oxide synthase-3 uncoupling stimulates cardiac pathologic remodeling from chronic pressure load. J Clin Invest 115: 1221-1231, 2005.

86. Goldberg LI and Sjoerdsma A: Effects of several monoamine oxidase inhibitors on the cardiovascular actions of naturally occurring amines in the dog. J Pharmacol Exp Ther 127: 212-218, 1959.

87. Zoccali C, Mallamaci F, Parlongo, et al: Plasma norepinephrine predicts survival and incident cardiovascular events in patients with end-stage renal disease. Circulation 105: 1354-1359, 2002 . 
88. Anand IS, Fisher LD, Chiang YT, et al: Changes in brain natriuretic peptide and norepinephrine over time and mortality and morbidity in the Valsartan Heart Failure Trial (Val-HeFT). Circulation 107: 1278-1283, 2003

89. Kotlaba D and Rybicki B: Norepinephrine level as a predictor of mortality after first myocardial infarction. J Clin Basic Cardiol 8: 55-58, 2005.

90. Otte C, Neylan TC, Pipkin SS, et al: Depressive symptoms and 24-hour urinary norepinephrine excretion levels in patients with coronary disease: findings from the Heart and Soul Study. Am J Psychiatry 162: 2139-2145, 2005.

91. Yanik Mand Erel O: The relationship between potency of oxidative stress and severity of depression. Acta Neuropsychiatrica 16 200-203, 2004

92. Bilici M, Efe H, Koroglu MA, et al: Antioxidative enzyme activities and lipid peroxidation in major depression: alterations by antidepressant treatments. J Affect Disord 64: 43-51, 2001.

93. Sarandol A, Sarandol E, Eker SS, et al: Major depressive disorder is accompanied with oxidative stress: short-term antidepressant treatment does not alter oxidative-antioxidative systems. Hum Psychopharmacol 22: 67-73, 2007.

94. Forlenza MJ and Miller GE: Increased serum levels of 8-hydroxy2'-deoxyguanosine in clinical depression. Psychosom Med 68: $1-7,2006$.

95. Hamilton CA, Miller WH, Al-Benna S, et al: Strategies to reduce oxidative stress in cardiovascular disease. Clin Sci (Lond) 106: 219-234, 2004.

96. Landmesser U and Harrison DG: Oxidant stress as a marker for cardiovascular events: Ox marks the spot. Circulation 104 2638-2640, 2001.

97. Rajagopalan S, Meng XP, Ramasamy S, et al: Reactive oxygen species produced by macrophage-derived foam cells regulate the activity of vascular matrix metalloproteinases in vitro. Implications for atherosclerotic plaque stability. J Clin Invest 98: 2572-2579, 1996.

98. Khairova RA, Machado-Vieira R, Du J, et al: A potential role for pro-inflammatory cytokines in regulating synaptic plasticity in major depressive disorder. Int J Neuropsychopharmacol 12: 561-578, 2009.

99. Floyd RA: Antioxidants, oxidative stress, and degenerative neurological disorders. Proc Soc Exp Biol Med 222: 236-245, 1999.

100. Whittemore ER, Loo DT, Watt JA, et al: A detailed analysis of hydrogen peroxide-induced cell death in primary neuronal culture. Neuroscience 67: 921-932, 1995.

101. Pasic J, Levy WC and Sullivan MD: Cytokines in depression and heart failure. Psychosom Med 65: 181-193, 2003.

102. Koenen RR and Weber C: Therapeutic targeting of chemokine interactions in atherosclerosis. Nat Rev Drug Discov 9: 141-153, 2010.

103. Ross R: Atherosclerosis - an inflammatory disease. N Engl J Med 340: 115-126, 1999.

104. Chaitidis P, Billett E, Kuban RJ, et al: Expression regulation of $\mathrm{MAO}$ isoforms in monocytic cells in response to Th2 cytokines. Med Sci Monit 11: 259-265, 2005.
105. Sherwood A, Hinderliter AL, Watkins LL, et al: Impaired endothelial function in coronary heart disease patients with depressive symptomatology. J Am Coll Cardiol 46: 656-659, 2005.

106. Musselman DL, Tomer A, Manatunga, et al: Exaggerated platelet reactivity in major depression. Am J Psychiatry 153: 1313-1317, 1996.

107. Musselman DL and Nemeroff CB: Depression really does hurt your heart: stress, depression, and cardiovascular disease. Prog Brain Res 122: 43-59, 2000.

108. Laghrissi-Thode F, Wagner WR, Pollock BG, et al: Elevated platelet factor 4 and beta-thromboglobulin plasma levels in depressed patients with ischemic heart disease. Biol Psychiatry 42: 290-295, 1997.

109. Pace TW and Miller AH: Cytokines and glucocorticoid receptor signaling. Relevance to major depression. Ann N Y Acad Sci 1179: 86-105, 2009.

110. Schins A, Honig A, Crijns H, et al: Increased coronary events in depressed cardiovascular patients: 5-HT2A receptor as missing link? Psychosom Med 65: 729-737, 2003.

111. Nemeroff CB and Musselman DL: Are platelets the link between depression and ischemic heart disease? Am Heart J 140: 57-62, 2000.

112.Ziegelstein RC, Parakh K, Sukhuja A, et al: Platelet function in patients with major depression. Intern Med J 39: 38-43, 2009.

113. Parissis JT, Farmakis D, Nikolaou M, et al: Plasma B-type natriuretic peptide and anti-inflammatory cytokine interleukin-10 levels predict adverse clinical outcome in chronic heart failure patients with depressive symptoms: a 1-year follow-up study. Eur J Heart Fail 11: 967-972, 2009.

114. Gillespie CF and Nemeroff CB: Hypercortisolemia and depression. Psychosom Med 67: S26-S28, 2005.

115. Gold PW, Wong ML, Goldstein DS, et al: Cardiac implications of increased arterial entry and reversible 24-h central and peripheral norepinephrine levels in melancholia. Proc Natl Acad Sci USA 102: 8303-8308, 2005.

116. Meltzer HY, Lowy MT and Locascio JJ: Platelet MAO activity and the cortisol response to dexamethasone in major depression. Biol Psychiatry 24: 129-142, 1988.

117. Slotkin TA, Seidler FJ and Ritchie JC: Effects of aging and glucocorticoid treatment on monoamine oxidase subtypes in rat cerebral cortex: therapeutic implications. Brain Res Bull 47: 345-348, 1998

118. Rosmond R and Bjorntorp P: The hypothalamic-pituitaryadrenal axis activity as a predictor of cardiovascular disease, type 2 diabetes and stroke. J Intern Med 247: 188-197, 2000.

119. Bugajski J, Turon M, Gadek-Michalska A, et al: Catecholaminergic regulation of the hypothalamic-pituitaryadrenocortical activity. J Physiol Pharmacol 42: 93-103, 1991.

120. Doyle A, Hucklebridge F, Evans P, et al: Urinary output of endogenous monoamine oxidase inhibitory activity is related to everyday stress. Life Sci 58: 1723-1730, 1996. 